\title{
Preprocessing TeCHNiQUes to IMPRove CNN BASED FACE RECOGNITION SYSTEM
}

\author{
Jayanthi Raghavan and Majid Ahmadi \\ Department of Electrical and Computer Engineering, \\ University of Windsor, Windsor, Canada
}

\begin{abstract}
In this work, deep CNN based model have been suggested for face recognition. CNN is employed to extract unique facial features and softmax classifier is applied to classify facial images in a fully connected layer of CNN. The experiments conducted in Extended YALE B and FERET databases for smaller batch sizes and low value of learning rate, showed that the proposed model has improved the face recognition accuracy. Accuracy rates of up to $96.2 \%$ is achieved using the proposed model in Extended Yale B database. To improve the accuracy rate further, preprocessing techniques like SQI, HE, LTISN, GIC and DoG are applied to the CNN model. After the application of preprocessing techniques, the improved accuracy of $99.8 \%$ is achieved with deep CNN model for the YALE B Extended Database. In FERET Database with frontal face, before the application of preprocessing techniques, CNN model yields the maximum accuracy of $71.4 \%$. After applying the above-mentioned preprocessing techniques, the accuracy is improved to $76.3 \%$
\end{abstract}

\section{KEYWORDS}

CNN, ANN, GPU

\section{INTRODUCTION}

For human brain, recognizing face is a very simple task and can be performed fast. In computer vision, face recognition is a very challenging task. Even though the face recognition research is in an advanced state [7], till now it is not possible to obtain results on par with humans.

To date, many approaches have been suggested for facial recognition. Holistic method works by projecting facial images onto a low-dimensional space, which neglects surplus details and variations that are not needed for the facial recognition [11]. One of the methods under this category is PCA [2]. The holistic methods are sensitive to local distortions like facial expression or illumination variation.

Subsequently, progress in the field of computer vision led to the growth of feature-based method in which features are extracted from various parts of a face image. Feature-based methods are robust to local variations such as intensity variation and face expression changes [22]. With the development of the local feature descriptors, feature based methods gained popularity. Local Binary Pattern (LBP) [6] is an extensively applied local feature descriptor in face recognition.

The recent trend is towards neural network-based approach [30]. Deep learning-based methods achieve excellent results in many fields like robotics and autonomous driving cars [3]. Deep learning methods are based on convolutional neural networks (CNNs). CNNs are slightly 
different from normal neural network. In $\mathrm{CNN}$, neurons in convolutional layer are thinly connected to the neurons in the next layer based on their relative location. CNNs are multilayer network trained from end to end with raw image pixel values assigned to classifier outputs.

The main advantage of deep learning methods is that they can be trained with very big datasets to learn the vital features to represent the input data. The main issue with deep learning method is models trained with small datasets are having the problem of poor generalization, which results in over-fitting.

Generalization term indicates the performance difference of a network model when assessed on earlier viewed training data against the testing data, the network has never viewed before [8]. Models with poor generalizability have overfitted the training data.

Overfittingis a term used when the network model functions extremely good with the training data, but could not work well with the test data. In the overfitted network validation error goes up while the training error comes down [21].

To reduce the overfitting, regularization process is employed on the network model. Regularization is a method of making minute changes to the actual network model and the learning algorithm, so that the model functions better in both training and testing data set. Regularization is defined as "Allowing to generalize well to unseen data even when training on a finite training set or with an imperfect optimization procedure" [4]. There are various regularization techniques available in machine learning like Dropout, Data Augmentation, Early stopping, batch normalization etc. [10]. Some of the regularization techniques are explained below.

Dropout means removing units temporarily in a neural network, together with all the incoming and outgoing links [23]. Dropout can be explained as the regularization technique by including noise to the hidden units of the network.

Another popular technique is batch normalization. Batch Normalization operates by deducting the batch mean from each activation and dividing by the standard deviation of the batch [8]. The normalization technique together with standardization is used as a typical combination in the preprocessing of pixel values. Batch normalization technique can be employed to any individual layer within the network.Hence it is powerful [5].

Data augmentation is an extensively applied technique used to artificially inflate the size of the training dataset by the methods called data warping or oversampling. The augmented data is a representation of detailed set of feasible data points, thus minimizing the distance between the training and the validation set.

The main aim of augmentation technique is to diminish the effect of overfitting on models using traditional transformations to manipulate the training data. As an example, a labeled image in the dataset can be increased by various processes like flipping, rotation, morphing and zooming. After some time, trained network gets exposure to such modifications and the it can identify the same object with different variations.

Optimizers update the weight parameters to minimize the cost function. Cost function is defined as the difference between predicted and actual output. One of the popular optimizers is Adam optimizer [1]. 
Adam is an adaptive learning rate optimization algorithm, which calculates learning rates for different parameters individually. Adam uses computations of first and second moments of gradient to adjust the learning rate for each weight of the network.

Application of regularization methods help to improve the accuracy rates. To increase the accuracy rates further, preprocessing techniques are applied to deep CNN architecture.

\section{Preprocessing Methods}

Face recognition task becomes challenging due to illumination conditions, occlusion, pose and facial expression variations [33]. The variation in illumination is one of the main challenging problems which affects the performance of the face recognition system. Among all, shadowing effect, underexposure, and overexposure conditions are challenging problems that need to be addressed in the face recognition process [35]. If the lighting conditions present in the gallery image is different from the probe image, then the process of face recognition may completely fail [34]. A good face recognition system should be able to give accurate recognition rate under the different illumination conditions between images of the same face [32]. Image enhancement algorithms play a great role in handling the illumination variation.

The main aim of preprocessing is to remove features that obstruct the process of classifying the images of the same person (within-class differences), thereby boosting the difference of them with others (between-class differences) [36].

For better face recognition under uncontrolled and illumination variation conditions, the vital features responsible for differentiating two different faces require to be retained. The shadows produced in facial images due to variation in lighting directions may cause loss of important facial features which are helpful for recognition. A preprocessing method must enhance the intensity in the regions of inadequately illuminated and decrease the intensity in the densely illuminated regions while retaining the intensity in the fairly illuminated portions [37]. Few important preprocessing techniques are discussed below.

\subsection{Histogram Equalization (HE)}

HE [38] flattens the histogram of the image and expands the dynamic range of the pixel intensity values by employing cumulative density function. The Histogram Equalization is a global preprocessing technique.

For image $\mathrm{I}(\mathrm{x}, \mathrm{y})$ with discrete $\mathrm{k}$. gray values histogram is defined by the probability of occurrence of the gray level I [39] is given by equation (1) as follows.

$$
\mathrm{p}(\mathrm{i})=n_{i} / \mathrm{N}
$$

Where $\mathrm{i} \in 0,1 \ldots \mathrm{k}-1$ grey level and $\mathrm{N}$ is total number of pixels in the image.

\subsection{Self Quotient Image (SQI)}

SQI [40] is one of the illumination invariant algorithm suggested for handling both shadow and lighting changes. It is defined as the ratio of the intensity of the input image to its smooth version as given in equations (2) and (3).

$$
\mathrm{Q}(\mathrm{x}, \mathrm{y})=\mathrm{I}(\mathrm{x}, \mathrm{y}) / \mathrm{S}(\mathrm{x}, \mathrm{y})
$$




$$
=\mathrm{I}(\mathrm{x}, \mathrm{y}) /(\mathrm{F}(\mathrm{x}, \mathrm{y}) * \mathrm{I}(\mathrm{x}, \mathrm{y}))
$$

where $I(x, y)$ is the face image and $S(x, y)$ is a smoothed version of the image and $*$ is the convolution operation. $\mathrm{F}$ is the smoothing kernel which in this case is a weighted Gaussian filter and Q is the Self Quotient Image since it is derived from one image and has the same quotient form as that in the quotient image method.

\subsection{Locally Tuned Inverse Sine Nonlinear (LTISN)}

LTISN [41] is a nonlinear and pixel by pixel approach, where the improved intensity values are calculated by applying the inverse sine function with a tunable parameter based on the nearby pixel values given in the equations (4), (5), (6), (7) and (8). The intensity range of the image is rescaled to $[01]$ followed by a nonlinear transfer function.

$$
\begin{gathered}
I_{\text {enh }}(x, y)=\frac{2}{\pi} \sin ^{-1}\left(I_{n}(x, y)^{q / 2}\right) \\
\operatorname{Kernel} l_{i}\left(n_{1}, n_{2}\right)= \\
\operatorname{kernel}_{i}\left(n_{1}, n_{2}\right)=\frac{h_{g}\left(n_{1}, n_{2}\right)}{\sum_{n_{1} \sum_{n_{2}} h_{g}\left(n_{1}, n_{2}\right)}} \\
h_{g}\left(n_{1}, n_{2}\right)=e^{\frac{-\left(n 1^{2}+n 2^{2}\right)}{2 \sigma^{2}}} \\
q=\left\{\begin{array}{l}
\tan \left(\frac{\pi}{C_{1}} I_{M_{n}}(x, y)\right)+C_{2} I_{M_{n}}(x, y) \geq 0.3 \\
\frac{1}{C_{3}} \ln \left(\frac{1}{0.3} I_{M_{n}}(x, y)\right)+C_{4} I_{M_{n}}(x, y)<0.3
\end{array}\right.
\end{gathered}
$$

\subsection{Gamma Intensity Correction (GIC)}

GIC [42] is a nonlinear gray-level transformation that substitutes gray-level I with the gray level $I^{\frac{1}{\square}}$, given by the equation (9).

$$
I=I^{\frac{1}{0}}
$$

As shown in the (Fig.1), for the values of gamma less than 1.0 darkens the image and for the values of gamma greater than 1.0 lightens the image. When gamma value is 1.0 , it does not produce any effect. 


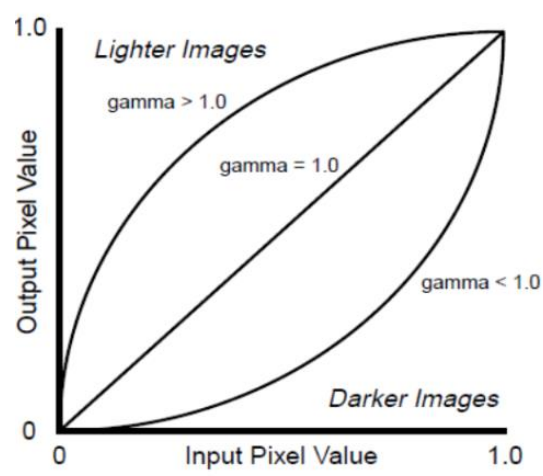

Fig.1. Gamma Intensity Correction

\subsection{Difference of Gaussian (DoG)}

DoG is a grayscale image enhancement algorithm [42] that involves the subtraction of one blurred version of an original grayscale image from another less blurred version of the original. The blurred images are obtained by convolving the original grayscale image with Gaussian kernels having differing standard deviations [34], which is given in the equation (10).

$$
\operatorname{DOG}(x, y)=\frac{1}{2 \pi \sigma_{1}^{2}} e^{-\frac{x^{2}+y^{2}}{2 \sigma_{1}^{2}}}-\frac{1}{2 \pi \sigma_{2}^{2}} e^{-\frac{x^{2}+y^{2}}{2 \sigma_{2}{ }^{2}}}
$$

$\sigma_{1}, \sigma_{2}$ are Gaussian kernel widths.

\subsection{Contrast-Limited Adaptive Histogram Equalization (CLAHE)}

CLAHE works on small areas in the image, known as tiles, rather than the whole image [43]. Individual tile's contrast is improved. Hence histogram of the output area is roughly matching with the histogram specified by the distribution parameter. The tiles present in the neighborhood regions are then joined by applying bilinear interpolation to minimize the effect of artificially induced border line [39]. In CLAHE, the image is divided into a limited number of regions and the same histogram equalization technique is applied to pixels in each region [44].

\section{Convolutional Neural Network}

Deep learning-based methods have shown better performances in terms of accuracy and speed of processing in image recognition.

\subsection{CNN Basics}

CNN is biologically inspired by visual cortex in brain [20]. Different phases of learning procedure in $\mathrm{CNN}$ is similar to the visual cortex. The visual cortex has small group of cells that are reactive to particular regions of the visual field. Hubel and Wiesel [17] experimentally showed that particular group of neurons in the cat's brain reacted to the appearance of edges of a certain orientation as shown in the (Fig.2). Further they illustrated that one specific set of neurons were activated, when exhibited to vertical edges and also another set of neurons fired when exposed to horizontal or diagonal edges. All of these categories of neurons are arranged in a columnar configuration and collectively these neurons can produce visual perception. 
Different portions of the visual cortex are classified as V1, V2, V3, and V4. In general, V1 and V2 regions of visual cortex are having close resemblance with convolutional and subsampling layers, whereas inferior temporal region resembles the higher layers of CNN [16]. During the process of training, CNN learns with the help of backpropagation algorithm by making adjustments in weights with respect to the target.

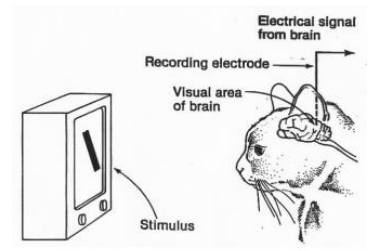

Fig.2. Hubel and Wiesel experiment

\subsection{CNN Architecture}

In $\mathrm{CNN}$ architecture, network layers are divided into three types: the convolutional, pooling and fully connected layers [20]. The architecture of CNN is as shown in Fig 3.

\subsubsection{Convolutional Layer}

In CNN, every neuron in the convolutional layer is linked only to a small portion of the neurons in the preceding layer, which is in square shape area across the height and width dimensions. The size of this square is a hyperparameter (controllable parameter) and called as Receptive Field. For the depth dimension, there is no hyperparameter available since the convolution operations are normally carried out for the whole depth. Generally, the depth dimension of the input describes the various colors of the image. Hence it is usually required to link them in order to bring out necessary information.

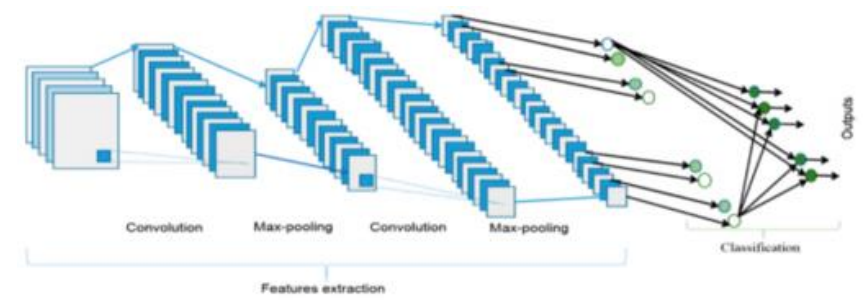

Fig.3.The CNN architecture

Neurons present in the convolution operator can recognize certain local patterns of the previous layer's output. Once features are obtained, its actual position is not important and all the neurons are expected to identify the same pattern. This is achieved by forcing all the neurons to have a common single set of parameters known as Parameter Sharing [9].

In order to identify various unique features within one layer, it is necessary to have multiple filters, where each filter is a group of neurons that recognize a certain pattern at different locations in the image. 


\subsubsection{Pooling Layer}

The main aim of pooling layer is to reduce the complexity of CNNs. The neurons present in the pooling layer form a square shaped area across the width and height dimensions of the preceding layer. Even though it is very similar to the convolutional layer, it is different from convolutional layer because the pooling layer is Non-ParametrizedLayer.

The function carried out by this layer is known as subsampling or down sampling. During this process, contraction in size results in concurrent loss of data. On the other hand, such a loss is helpful tothe network because the reduction in size not only reduces the computational burden for the succeeding layers of the network and also it reduces the effects of overfitting [12].

Max pooling and average pooling are the generally used techniques shown in (Fig.4). Max Pooling chooses the largest element within each receptive field [14] whereas Average Pooling computes the average among the output neurons within the pooling window. Max-pooling chooses the most prominent feature in a pooling window. On the other hand, average-pooling method selects whole features into consideration. Thus, max-pooling method keeps texture related information, while average pooling method retains the background related data [24]. Pooling operation does not combine neurons with different depth values. Instead, the resulting pooling layer will have the uniform depth as the previous layer and it will only combine local areas within a filter.

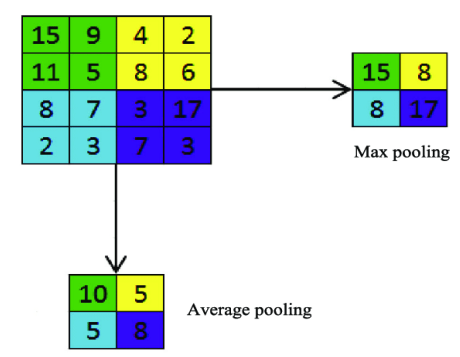

Fig.4. Max Pooling and Average Pooling Operations Illustration.

\subsubsection{Fully Connected Layer}

The filters and neurons present in this layer are connected to all the activation in the preceding layers resulting in a completely connected structure. Hence the name. The output feature maps of the final convolution or pooling layer is converted into a one-dimensional (1D) array of numbers [18]. High-level reasoning in the network is carried out via fully connected layers [19].

The final fully connected layer has the same number of output nodes as the number of classes. Each fully connected layer is followed by a nonlinear function, such as ReLU (Rectified Linear Units). $R e L U$ is an activation function operates by thresholding values at 0 , i.e. $\mathrm{f}(\mathrm{x})=\max (0, \mathrm{x})$. In other words, it outputs 0 when $x<0$, and contrarily, it outputs a linear function with a slope of 1 when $x \geq 0$ [15]as shown in the (Fig.5). 


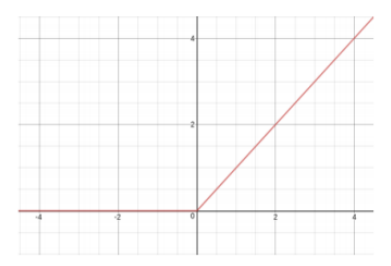

Fig.5. The Rectified Linear Unit (ReLU)

\subsection{CNN Operation}

Based on local receptive field, each component in a convolutional layer accepts inputs from a set of adjacent units belonging to the preceding layer layer. This way neurons are proficient in extracting rudimentary features like edges or corners. These features are then linked by the succeeding convolutional layers in order to further extract high level features.

The components of a convolutional layer are arranged in planes. All units of a plane share the same set of weights. Thus, each plane is in charge for building a particular feature. The results obtained from the plane is termed as feature maps. Each convolutional layer consists of several planes, so that multiple feature maps can be constructed at each location. The most significant features derived are passed from initial layers to higher layers. As the features are passed to the higher layer, there is a dimensionality reduction in features determined by kernel size of the convolutional and max-pooling layers. On the other hand, there is an increase in number of feature maps for representing better features of the input images for ensuring classification accuracy [13]. The derived feature vector either could be an input for classification task or could be treated as a feature vector for next level processing.

The network designed to do the classification task consists of several convolution pooling layers followed by some fully-connected layers. The first two layers mentioned above perform convolution and pooling operation in order to extract high-level features.The final layer's output of CNN is applied as the input to a fully connected network, which does the task of classification.

The output layer for classification task consists of one neuron for each class and the values of these neurons describes the score of each class. If score distribution is chosen, the score range will be between zero and one. The summation of class scores is one. The values of each neuron can be presumed as the probability of occurrence of the class.

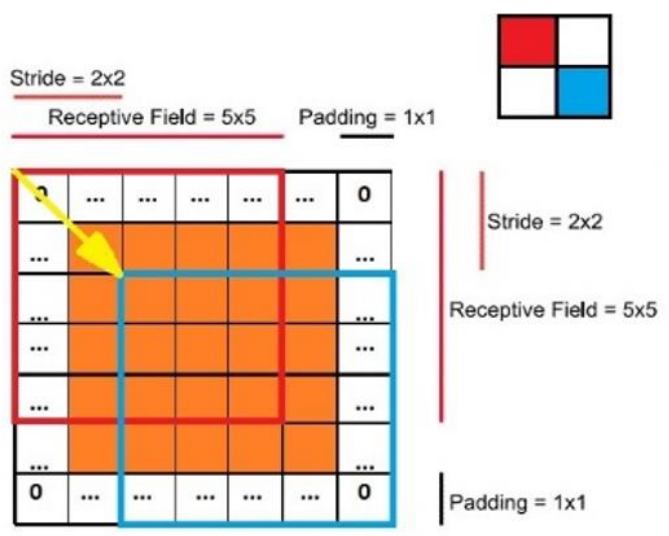

Fig.6. A visual representation of the various hyperparameters of convolutional layers: receptive field, stride and padding 


\section{EXPERIMENTAL SETUP}

The implementations are carried out in MATLAB 2019b on a workstation with windows 10 OS, AMD processor with $2.0 \mathrm{GHz}$, hard drive with $8 \mathrm{~GB}$ RAM. The experiments are conducted in Extended Yale B and FERET Database with deep CNN model.

\subsection{Extended Yale B Database}

The extended Yale Face Database B contains 16128 images of 28 human subjects under 9 poses and 64 illumination conditions as shown in (Fig.7). The data format of this database is the same as that of the Yale Face Database B.

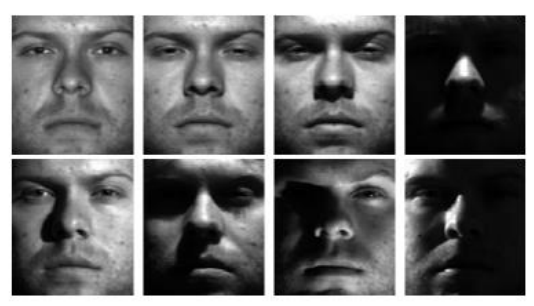

Fig.7. Images from extended Yale B database.

\subsection{FERET Database}

The FERET database was collected in 15 sessions between August 1993 and July 1996. The database contains 1564 sets of images for a total of 14,126 images that includes 1199 individuals and 365 duplicate sets of images as shown in (Fig.8). A duplicate set is a second set of images of a person already in the database and was usually taken on a different day.

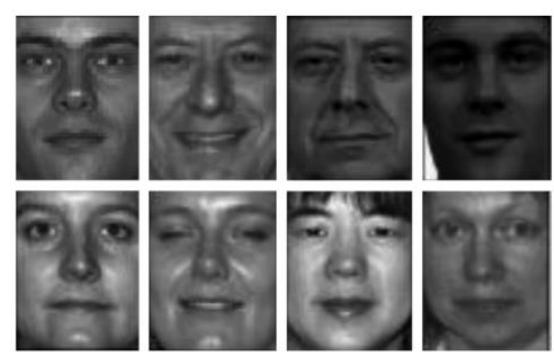

Fig.8. Images from FERET database

\subsection{Experiment}

The model uses a deep CNN network with six convolution layers (with size 3x3), two maxpooling and two fully convolutional layers. The first two convolutions use 8 filters, next two uses 16 and the last convolution layers uses 32 each in order to extract complex features.

The model uses batch normalization and dropouts in all convolutional layers along with ReLU activation function. In order to reduce the overfitting, the dropout rate is kept higher on the final convolutional layers. Two maxpooling layers are introduced after second and fourth convolutional layers to reduce the feature space dimension. The two fully connected layers use 128 and 28 neurons respectively. The final classification layer uses softmax activation with 
categorical cross entropy loss function. The softmax layer is used to produce the classification scores, in which each score is the probability of a particular class for a given instance [31].

The dropout principle is employed on the convolutional neural networks model and the value of drop out is chosen by trial and error method. The (Fig.9) shows the network model with drop out and the values of the drop out is different for different layers. The model also uses Adam optimizer with a learning rate of 0.001 which was found empirically after trying different combinations.

The feature maps are subsampled with maxpooling layers with a stride of $2 \times 2$. Stride is the number of pixels which shifts over the input matrix. When the stride is 2 , it means the filter is moved by 2 pixels at a time. The number of neurons in this output layer is limited to 28 in Extended Yale B database. In FERET database, the number of neurons in the output layer is 994.

The training is designed to use different batch sizes to do a fair trade off between accuracy and training time.

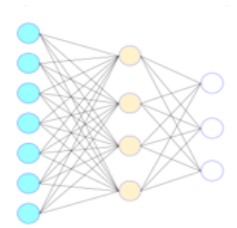

(a)

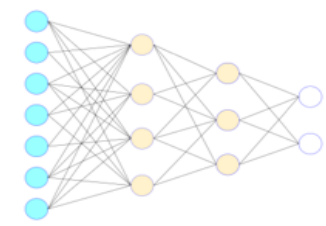

(b)

Fig.9. (a) The neural networks model (b) The model after applying drop out

\section{RESULTS}

The CNN trained with back-propagation algorithm in batch mode with a various batch sizes of 4 , 8, 16 and 32 for different epochs are computed for Extended YALE B with an image size of $128 \times 128$ for 50 epochs. The same experiment is conducted on FERET database for the frontal face image size of $128 \times 128$ for 300 epochs.

For the Extended YALE B Database, the maximum accuracy rate of $97.2 \%$ is achieved for the batch size of 4 without applying preprocessing technique as shown in (Fig.10). After applying the preprocessing techniques, the maximum accuracy is improved to $99.8 \%$ as shown in (Fig.11).

For the batch size of 8 , the maximum accuracy rate of $97.0 \%$ is achieved without applying any preprocessing technique. After applying the preprocessing techniques, the maximum accuracy is improved to $99.4 \%$.

For the batch size of 8, SQI, LTISN and HE perform very equally well and yield the maximum accuracy of $99.4 \%, 99.3 \%$ and $99.1 \%$.

For the batch size of 16 , the maximum accuracy rate of $96.8 \%$ is achieved without applying preprocessing technique. After applying the preprocessing techniques, the maximum accuracy is improved to $99.1 \%$.

For the batch size of $16 \mathrm{HE}, \mathrm{SQI}$ and GIC perform equally well and yield the maximum accuracy of $99.1 \% .98 .8 \%$ and $98.8 \%$ respectively. 
For the batch size of 32 , the maximum accuracy rate of $96.2 \%$ is achieved without applying preprocessing technique. After applying the preprocessing techniques, the maximum accuracy is improved to $98.7 \%$ for the same batch size.

For the batch size of 32, GIC, SQI, and HE perform equally well and yield the maximum accuracy of $98.7 \%, 98.3 \%$ and $98.3 \%$ respectively. Table 1 shows the accuracy rates of Extended Yale B database for various batch sizes before and after application of preprocessing techniques.

For the FERET Database, the maximum accuracy rate of $71.4 \%$ is achieved without applying preprocessing technique, for the batch size of 4, as shown in (Fig.12). After applying the preprocessing techniques, the maximum accuracy improved to $76.6 \%$ as shown in (Fig.13).

DoG, HE and LTISN perform equally well and yield the maximum accuracy of $76.6 \%, 76.3 \%$ and $75.6 \%$ respectively.

For the batch size of 8 , the maximum accuracy rate of $71.2 \%$ is achieved without applying preprocessing technique. After applying the preprocessing techniques, the maximum accuracy is improved to $76.4 \%$.

For the batch size of $8, \mathrm{HE}, \mathrm{SQI}$ and LTISN perform very equally well and yield the maximum accuracy of $76.4 \%, 74.6 \%$ and $72.5 \%$ respectively.

For the batch size of 16 , the maximum accuracy rate of $71.0 \%$ is achieved without applying preprocessing technique. After applying the preprocessing techniques, the maximum accuracy is improved to $76.1 \%$.

For the batch size of $16 \mathrm{HE}$, LTISN and SQI perform very equally well and yield the maximum accuracy of $76.1 \%, 72.0 \%$ and $71.5 \%$ respectively.

For the batch size of 32 , the maximum accuracy rate of $68.7 \%$ is achieved without applying preprocessing technique. After applying the preprocessing techniques, the maximum accuracy is improved to $71.0 \%$.

For the batch size of 32, SQI, GIC, LTISN and DoG perform equally well and yield the maximum accuracy of $71 \%, 70.9 \%, 70.8 \%$ and $69.9 \%$ respectively.

SQI and HE perform equally well and provide best results for all the batch sizes in both Extended Yale $\mathrm{B}$ and FERET database.

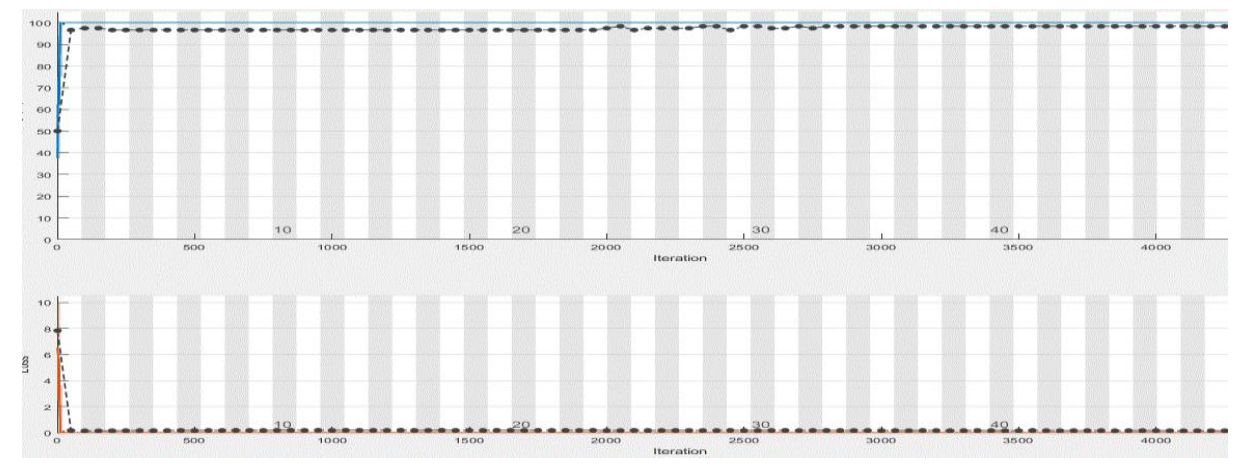

Fig.10.Accuracy using Extended YALE B Database before applying of preprocessing techniques 


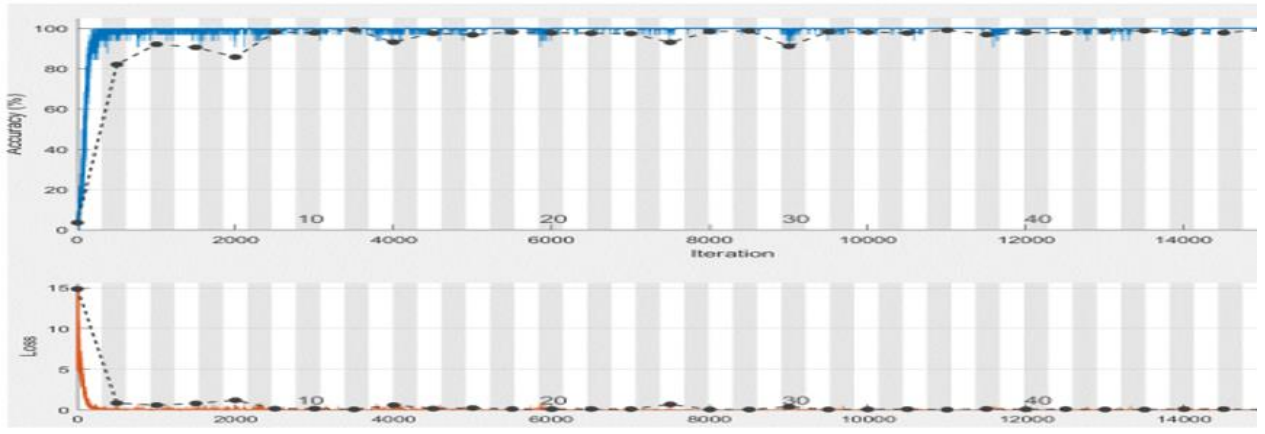

Fig.11. Accuracy using Extended YALE B Database after application of preprocessing techniques

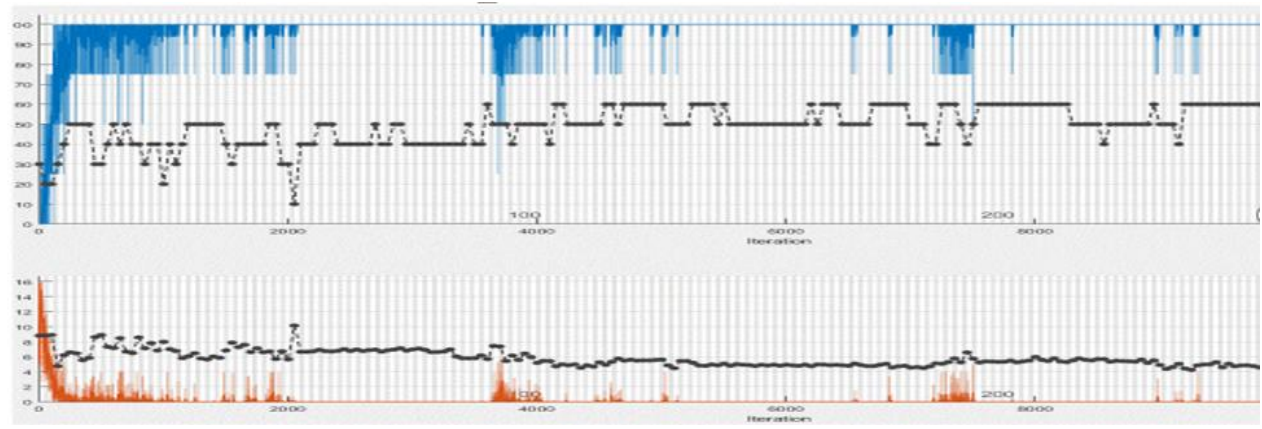

Fig.12. Accuracy using FERET Database before the application of pre processing techniques.

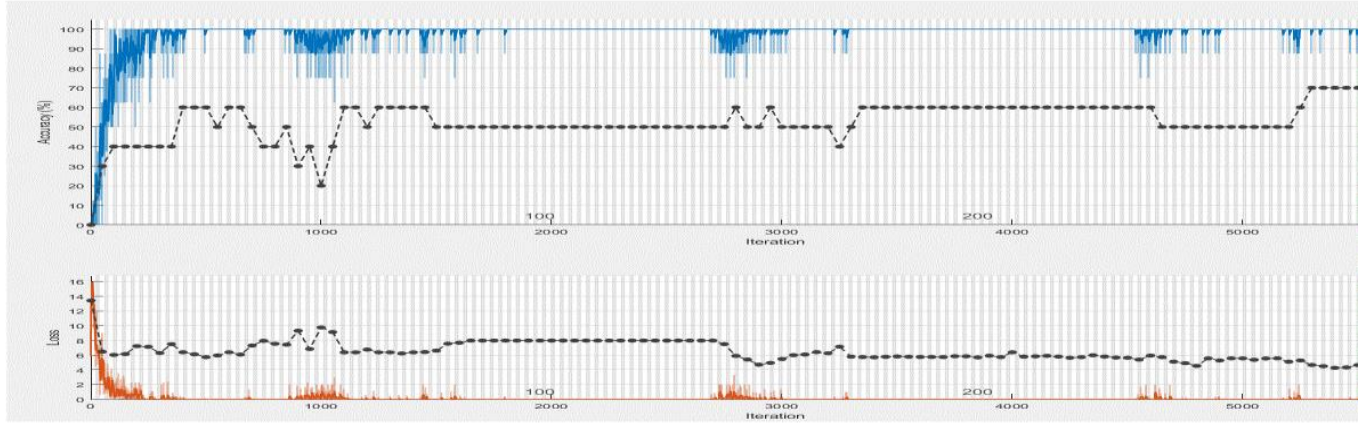

Fig.13. Accuracy using FERET Database after application of pre processing techniques.

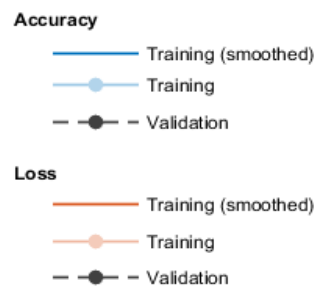


Table 1 Accuracy rates of Extended Yale B database for various batch sizes before and after application of preprocessing techniques.

\begin{tabular}{|c|c|c|c|c|}
\hline \multirow{2}{*}{ Database } & \multicolumn{4}{|c|}{ Accuracy Rates } \\
\cline { 2 - 5 } & \multicolumn{4}{|c|}{ Batch Size } \\
\cline { 2 - 5 } & $\mathbf{4}$ & $\mathbf{8}$ & $\mathbf{1 6}$ & $\mathbf{3 2}$ \\
\hline $\begin{array}{c}\text { Extended Yale B } \\
\text { (WOAPP) }\end{array}$ & 97.2 & 97.0 & 96.8 & 96.2 \\
\hline DoG & & & & \\
\hline SQI & 99.1 & 99.0 & 98.7 & 97.9 \\
\hline LTISN & 99.8 & 99.4 & 98.8 & 98.3 \\
\hline HE & 99.2 & 99.3 & 98.4 & 98.1 \\
\hline GIC & 99.7 & 99.1 & 99.1 & 98.3 \\
\hline
\end{tabular}

Table 2 Accuracy rates of FERET database for various batch sizes before and after application of preprocessing techniques

\begin{tabular}{|l|l|l|l|l|}
\hline \multirow{2}{*}{ Database } & \multicolumn{4}{|c|}{ Bccuracy Rates } \\
\cline { 2 - 5 } & \multicolumn{3}{|c|}{ Batch Size } & \multirow{3}{*}{$\mathbf{3 2}$} \\
\cline { 2 - 5 } & $\mathbf{4}$ & $\mathbf{8}$ & $\mathbf{1 6}$ & 68.7 \\
\hline $\begin{array}{l}\text { FERET } \\
\text { (WOAPP) }\end{array}$ & 71.4 & 71.2 & 71 & 69.9 \\
\hline DoG & & & 71.3 & 71.0 \\
\hline SQI & 76.6 & 72.6 & 71.5 & 70.8 \\
\hline LTISN & 74.9 & 74.6 & 72.0 & 69.2 \\
\hline HE & 75.6 & 72.5 & 76.1 & 70,9 \\
\hline GIC & 76.3 & 76.4 & 71.0 & \\
\hline
\end{tabular}

\subsection{Number of trainable parameters calculation in Model}

The number of trainable parameters is computed for each layer as below. The deep architecture is as shown in (Fig.14).

\section{$\mathrm{C}_{1}$ Layer}

Input image $128 \times 128 \times 1$ and having one channel.

Number of weights per filter $=3 \times 3 \times 1$

There are totally eight filters in $\mathrm{C} 1$ layer and there is one bias parameter for each filter.

Total number of trainable parameters in C1 Layer is $=(3 \times 3 \times 1+1) \times 8=80$.

\section{$\mathrm{C}_{2}$ Layer}

Input feature maps to $\mathrm{C}_{2}$ layer having 8 channels.

Number of weights per filter is $3 \times 3 \times 8$.

There are 8 such filters in the layer. Hence total number of weights $=((3 \times 3 \times 8)+1) \times 8$ and there is one bias for each filter.

Total weights in $\mathrm{C}_{2}=((3 \times 3 \times 8)+1) \times 12=584$. 


\section{$S_{1}$ Layer}

$\mathrm{S}_{1}$ layer is using max pooling operation. Hence no trainable parameter available for this layer.

\section{$\mathrm{C}_{3}$ Layer}

Input to $\mathrm{C}_{3}$ has eight feature channels.

Number of weights per filter is $3 \times 3 \times 8$.

There are 16 such filters in the layer. Hence total number of weights $=((3 \times 3) \times 8+1) \times 16$ and there is one bias for each filter.

Total weights in $\mathrm{C}_{3}=(3 \times 3 \times 8+1) \times 16=1168$.

\section{$\mathrm{C}_{4}$ Layer}

Input to $\mathrm{C}_{4}$ has sixteen feature channels.

Number of weights per filter is $3 \times 3 \times 16$.

There are 16 such filters in the layer. Hence total number of weights $=((3 \times 3) \times 16+1) \times 16$ and there is one bias for each filter.

Total weights in $\mathrm{C} 4=(3 \times 3 \times 16+1) \times 16=2320$.

\section{$\mathbf{S}_{2}$ Layer}

$\mathrm{S}_{2}$ layer is using max pooling operation. Hence no trainable parameter available for this layer.

\section{$\mathrm{C}_{5}$ Layer}

Input to $\mathrm{C}_{5}$ has sixteen feature channels.

Number of weights per filter is $3 \times 3 \times 16$.

There are 32 such filters in the layer. Hence total number of weights $=((3 \times 3) \times 16+1) \times 32$ and there is one bias for each filter.

Total weights in $\mathrm{C}_{4}=(3 \times 3 \times 16+1) \times 32=4640$. 

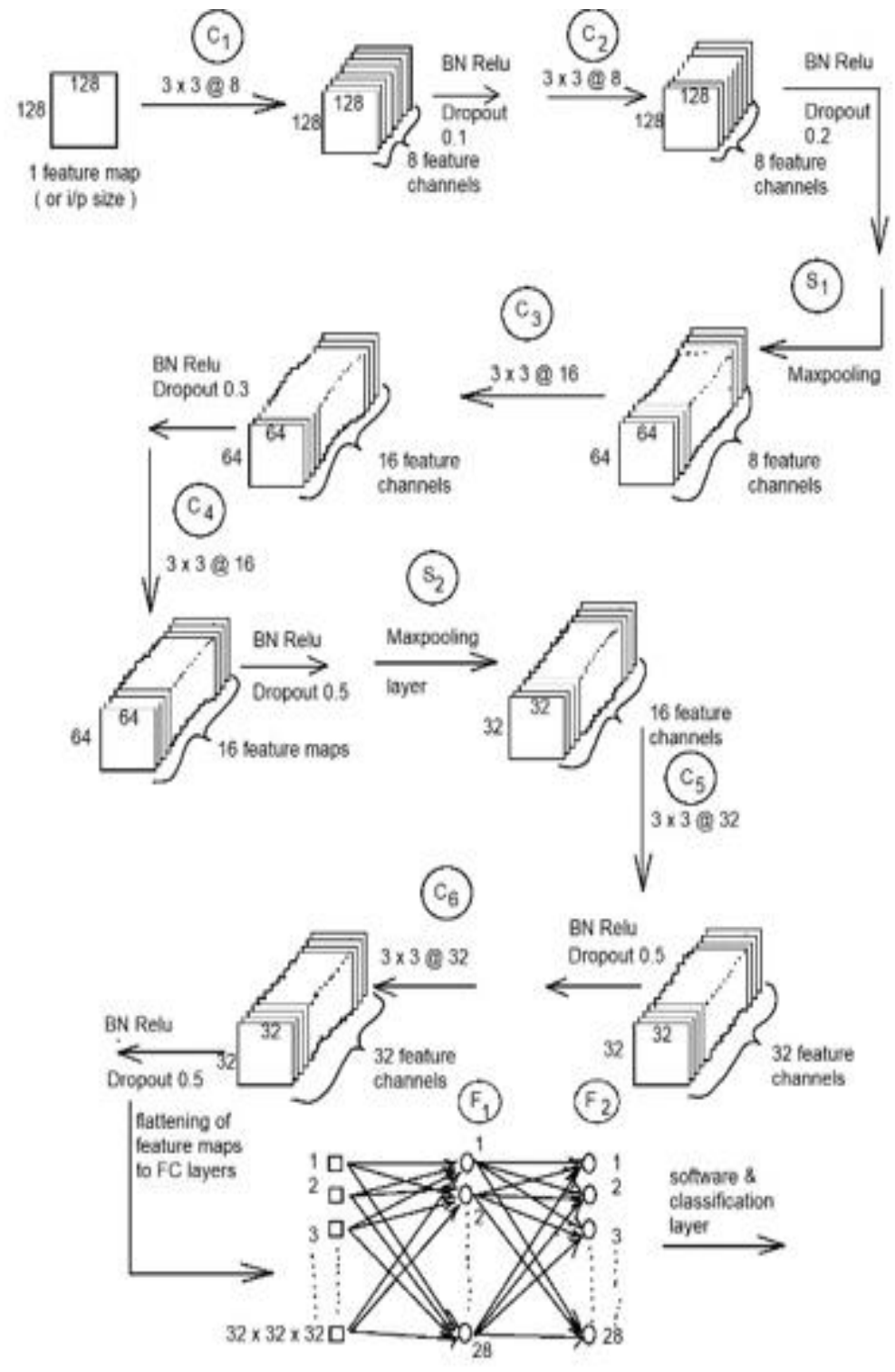

Fig.14. Deep CNN Model Architecture (BN-Batch Normalization)

\section{C6 Layer}

Input to $\mathrm{C}_{6}$ has 32 feature channels.

Number of weights per filter is $3 \times 3 \times 32$

There are 32 such filters in the layer. Hence total number of weights $=(3 \times 3 \times 32+1) \times 32$ and there is one bias for each filter.

Total weights in $\mathrm{C}_{4}=(3 \times 3 \times 32+1) \times 32=9248$.

\section{F $_{1}$ Layer}

In the fully connected layer, the input is having total feature space of size $32 \times 32$ and 32 . Input samples to $\mathrm{F}_{1}$ layer $=32 \times 32 \times 32$

There are 128 neurons in $F_{1}$ layer and each have a bias.

Total Number of trainable parameters for $F_{1}$ layer is $=(32 \times 32 \times 2+1) \times 128=4194432$. 


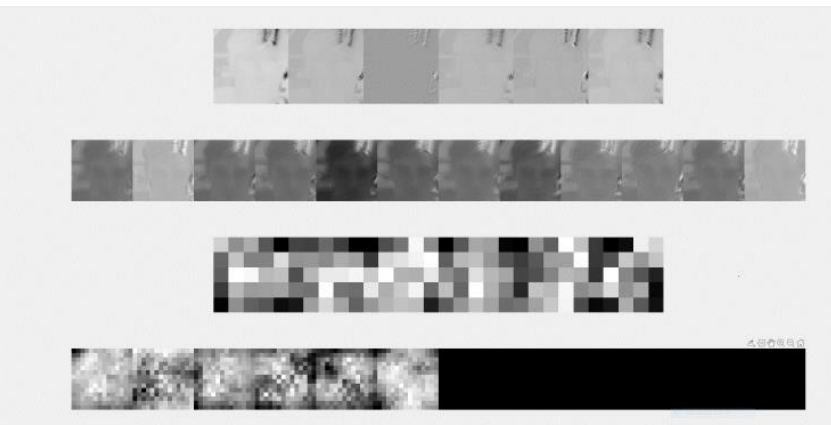

Fig.15.Feature map obtained for the batch size 32 and epoch 2

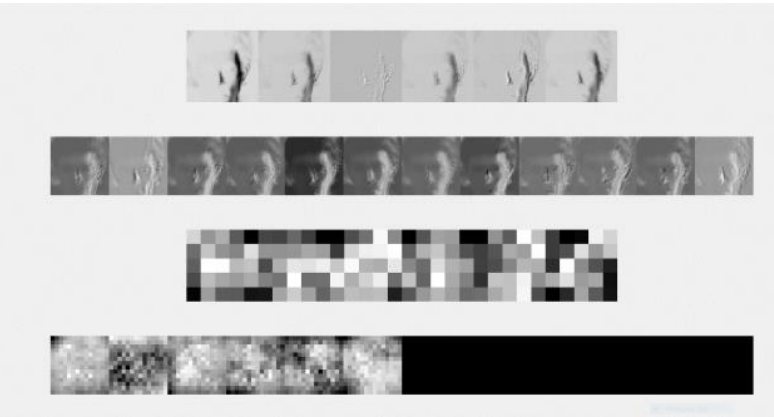

Fig.16.Feature map obtained for the batch size 32 and epoch 5

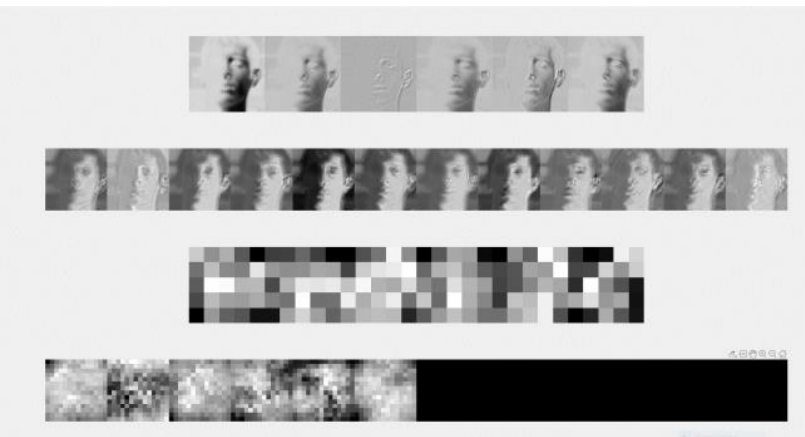

Fig.17.Feature map obtained for the batch size 32 and epoch 25

\section{F $_{2}$ Layer}

The input to $\mathrm{F}_{2}$ layer is the output from $\mathrm{F}_{1}$ layer. It is connected to 28 neurons (since there are 28 classes). So, total number of trainable parameters $=(128+1) \times 28=16512$.

Feature maps extracted for different batch size and epochs are as shown in the (Fig.15,16,17).

\section{COMPARISION With OTHER APPROACHES}

In this section, the proposed approach is compared with the various approaches.

Hybrid system, which combines convolutional neural network (CNN) and a Logistic regression classifier (LRC) yields the accuracy of $80 \%$. A CNN is trained to recognize facial images and 
LRC is used to classify the features learned by the convolutional network [26]. The neural network with the nonlinear theories, such as wavelet theory and fuzzy set is a new research idea, when applied in face recognition, gives accuracy around 93\% in Wavelet-BP [25]. CNN architecture along with Max-Feature-Map (MFM) could extract compact vital information yields the accuracy rate of 98\% [28]. The popular LeNet-5 architecture in FERET database yields accuracy rate is $81.25 \%$ [27]. The pre-trained CNN model along with the VGG-Face provides face verification rate accuracy of $86.85 \%$ and $83.32 \%$ on FRGC and LFW databases respectively [29].

Table 3. Comparison of Accuracy rates of different approaches.

\begin{tabular}{|l|l|l|l|}
\hline Sno & $\begin{array}{l}\text { Architecture } \\
\text { used }\end{array}$ & Dataset & Accuracy \\
\hline 1 & $\begin{array}{l}\text { Proposed } \\
\text { method } \\
\text { (CNN) }\end{array}$ & $\begin{array}{l}\text { Extended Yale B } \\
\text { FERET }\end{array}$ & $\begin{array}{l}\mathbf{9 9 . 8 \%} \\
\mathbf{7 6 . 3 \%}\end{array}$ \\
\hline 2 & $\begin{array}{l}\text { VGG+CNN } \\
{[29]}\end{array}$ & FRGC, LFW & $\begin{array}{l}\mathbf{8 6 . 8 5 \%} \\
\mathbf{8 3 . 3 2 \%}\end{array}$ \\
\hline 3 & $\begin{array}{l}\text { CNN with } \\
\text { MAF } \\
\text { activation } \\
\text { function [28] }\end{array}$ & LFW & $\mathbf{9 8 \%}$ \\
\hline 4 & $\begin{array}{l}\text { Wavelet-BP } \\
{[25]}\end{array}$ & AT\&T & $\mathbf{9 3 . 0 \%}$ \\
\hline 5 & $\begin{array}{l}\text { CNN-LRC } \\
{[26]}\end{array}$ & Yale & $\mathbf{8 0 \%}$ \\
\hline 6 & $\begin{array}{l}\text { CNN-LENET } \\
{[27]}\end{array}$ & FERET & $\mathbf{8 1 . 2 5 \%}$ \\
\hline
\end{tabular}

The proposed method compared with existing approaches are given in Table 3. The proposed method performs better in comparison to all other approaches in Extended Yale B. In FERET database, CNN-LENET method performs better than the proposed method.

\section{Conclusion}

In this paper, deep convolution neural network (CNN) is applied for extracting features and classification. The performance of the network is assessed before and after applying preprocessing techniques for various batch sizes and epochs on YALE B and FERET dataset.

Adam optimizers with a learning rate of 0.001 is applied in this experiment. The batch sizes used in this experiment are $[4,8,16,32]$;

The optimum batch sizes are chosen using trial and error method. Initially, multiple batch sizes of $[4,8,16,32,64,128]$ are selected for this experiment. Batch size of 4 with learning rate of 0.001 and number of epochs 50 give the best results. Batch sizes of $[4,8,16,32]$ provide consistent and best results. The batch sizes are normally selected in power of 2 . Choosing smaller batch size with low value of learning rate yields best result.

The results showed the maximum accuracy rate of $97.2 \%$ of achieved without using preprocessing techniques. When the preprocessing techniques are applied, the improved accuracy rates are achieved up to $99.8 \%$. 
The same experiments are conducted in FERET. dataset also. For the frontal face, the maximum $71.4 \%$ is achieved without optimization. After applying the preprocessing techniques, the accuracy rate increased to $76.3 \%$.

The proposed approach performs very well as compared to existing methods.

\section{REFERENCE}

[1] S. Setiowati, Zulfanahri, E. L. Franita and I. Ardiyanto, "A review of optimization method in face recognition: Comparison deep learning and non-deep learning methods" 9th International Conference on Information Technology and Electrical Engineering (ICITEE), Phuket, pp. 1-6, 2017.

[2] Aleix M. Marttinez and Avinash C. Kak, "PCA versus LDA".IEEE Transactions on Pattern Analysis and Machine Intelligence, Vol. 23, No.2, pp. 228-233, 2001.

[3] Rahul Haridas, Jyothi R L “Convolutional Neural Networks: A Comprehensive Survey” International Journal of Applied Engineering Research Volume 14, Number 3, pp. 780-789, (2019).

[4] Goodfellow, I. J., Bengio, Y., and Courville, A. Deep Learning MIT Press, (2016).

[5] Jason Wang, Luis Perez The Effectiveness of Data Augmentation in Image Classification using Deep Learning. In: Stanford University research report, 2017.

[6] Di Huang, Caifeng Shan, Mohsen Ardebilian, Yunhong Wang, and Liming Chen "Local Binary Patterns and Its Application to Facial Image Analysis: A Survey" IEEE Transactions on Systems, Man and Cybernetics, part C, volume 41, pp 765-781, 2011.

[7] Shailaja A Patil and Dr. P. J. Deore "Face Recognition: A Survey" Informatics Engineering, an International Journal (IEIJ), Vol.1, No.1, December 2013.

[8] Connor Shorten and Taghi M. Khoshgoftaar "A survey on Image Data Augmentation for Deep Learning" Jounal of Big Data, - Springer, 2019.

[9] Felix Ellenberger, Claus Lenz "A Non-Technical Survey on Deep Convolutional Neural Network Architectures" Computer Vision and Pattern Recognition, 2018.

[10] S.V.G. Reddy, K. Thammi Reddy, V. ValliKumari "Optimization of Deep Learning using various Optimizers, Loss functions and Dropout" International Journal of Recent Technology and Engineering (IJRTE) Volume-7 Issue-4S2, pp 448-455, December 2018.

[11] Saez Trigueros, D.; Meng, L.; Hartnett, M. Face Recognition: From Traditional to Deep Learning Methods. J. Theory. Appl. Inf. Technol. 2018, 97, 3332-3342.

[12] Athanasios Voulodimos, Nikolaos Doulamis, Anastasios Doulamis, and Eftychios Protopapadakis "Deep Learning for Computer Vision: A Brief Review" Computational Intelligence and Neuroscience, pp 1-13, 2018.

[13] Md Zahangir Alom, Tarek M. Taha, Chris Yakopcic, Stefan Westberg, Paheding Sidike, Mst Shamima Nasrin, Mahmudul Hasan, Brian C. van Essen, Abdul A. S. Awwal and Vijayan K. Asari "A State-of-the-Art Survey on Deep Learning Theory and Architectures". Electronics 2019, 8, 292

[14] Waseem Rawat and Zenghui Wang "Deep Convolutional Neural Networks for Image Classification: A Comprehensive Review" Volume 29, pp 2352-2449 September 2017.

[15] A. F. Agarap. Deep learning using rectified linear units (relu). CoRR, abs/1803.08375, URL http://arxiv.org/abs/1803.08375, 2018.

[16] Khan, Asifullah \& Sohail, Anabia \& Zahoora, Umme \& Saeed, Aqsa “A Survey of the Recent Architectures of Deep Convolutional Neural Networks", published in Artificial Intelligence Review (2019).

[17] D. H. Hubel and T. N. Wiesel. "Receptive fields of single neurons in the cat's striate cortex" The Journal of Physiology, 148(3):574-91, 1959.

[18] Yamashita, R., Nishio, M., Do, R.K.G. et al. "Convolutional neural networks: an overview and application in radiology” Insights Imaging 9, pp611-629, 2018.

[19] Mamoun Alazab, MingJian Tang "Deep Learning Applications for Cyber Security”, Springer, ISBN 978-3-030-13057-2, May 2019.

[20] N. Aloysius and M. Geetha, "A review on deep convolutional neural networks," 2017 International Conference on Communication and Signal Processing (ICCSP), Chennai, pp. 0588-0592, doi: 10.1109/ICCSP.2017.8286426, 2017. 
[21] Nusrat, Ismoilov and Sung-Bong Jang. "A Comparison of Regularization Techniques in Deep Neural Networks." Symmetry 10 (2018): 648.

[22] B. S. Manjunath, R. Chellappa and C. von der Malsburg, "A feature-based approach to face recognition," Proceedings 1992 IEEE Computer Society Conference on Computer Vision and Pattern Recognition, Champaign, IL, USA, pp. 373-378, 1992.

[23] Srivastava, Nitish et al. "Dropout: a simple way to prevent neural networks from overfitting." $J$. Mach. Learn. Res. 15, pp 1929-1958, (2014).

[24] Zhao, Qi et al. "Multiactivation Pooling Method in Convolutional Neural Networks for Image Recognition." Wireless Communications and Mobile Computing (2018): n. page. Wireless Communications and Mobile Computing. Web, 2018.

[25] J. Kittler, P. Koppen, et. al, "Conformal Mapping of a 3D Face Representation onto a 2D Image for CNN Based Face Recognition," 2018 International Conference on Biometrics (ICB), Gold Coast, QLD, pp. 124-131, 2018.

[26] H. Khalajzadeh, M. Manthouri and M. Teshnehlab, 'Face recognition using convolutional neural networks and simple logistic classifier', Advances in intelligent systems and computing, 223, pp 197207, (2014).

[27] A.R. syafeeza, M.K. Hani, S.S. Uiw and R. Bakhteri,' Convolutional neural network for face recognition with pose and illumination variation', International journal of engineering and Technology, 6(1), pp 44-57, (2014).

[28] Y. Xu, F. Liang, G. Zhang and H. Xu, 'Image intelligent detection based on the Gabor wavelet and the neural network', Symmetry, 8(130), (2016).

[29] Wu, Xiang et al. “A Lightened CNN for Deep Face Representation.” ArXiv abs/1511.02683 (2015): n. pag.

[30] Z. Lu, X. Jiang and A. Kot, "Enhance deep learning performance in face recognition," 2nd International Conference on Image, Vision and Computing (ICIVC), Chengdu, pp. 244-248, 2017.

[31] Pouyanfar, S., Sadiq, S., Yan, Y., Tian, H., Tao, Y., Reyes, M. P, Iyengar, S. S. A survey on deep learning: Algorithms, techniques, and applications. ACM Computing Surveys, 51(5), (2018).

[32] Y. Admi, Y. Moses and S. Ulman "Face Recognition: The problem of compensating for changes in illumination direction", IEEE Transaction. Pattern Anal. Mach. Intell., vol. 19, pp 721-732, Jul. 1997.

[33] Soodeh Nikan and M. Ahmadi. "Human Face Recognition under Occlusion using LBP and Entropy Weighted Voting” 21st International Conference on Pattern Recognition ICPR pp. 1699-1702, 2012.

[34] S. Anila, K. Arulsukanya, K. Divya, C. Kanimozhi, and D. Kavitha "An efficient Preprocessing Technique for Face Recognition under difficult Lighting Conditions," in Proc. National Conference on Emerging Trends in Computer Communication and Informatics (ETCCI-2011), March 10-11, 2011.

[35] Hu Han, Shiguang Shan, Xilin Chen, Wen Gao "A comparative study on illumination preprocessing in face recognition” Pattern Recognition., Volume 46, Issue 6, Pages 1691-1699, June 2013.

[36] Calvo G., Baruque B., Corchado E. (2013) Study of the Pre-processing Impact in a Facial Recognition System. In: Pan JS., Polycarpou M.M., Woźniak M., de Carvalho A.C.P.L.F., Quintián H., Corchado E. (eds) Hybrid Artificial Intelligent Systems. HAIS, Lecture Notes in Computer Science, vol 8073. Springer, Berlin, Heidelberg, 2013.

[37] Koringa P.A., Mitra S.K., Asari, V.K. Handling Illumination Variation: A Challenge for Face Recognition. In: Raman B., Kumar S., Roy P., Sen D. (eds) Proceedings of International Conference on Computer Vision and Image Processing. Advances in Intelligent Systems and Computing, vol 460. Springer, Singapore, 2017.

[38] Dr. A. Sri Krishna, G. Srinivasa Rao and M. Sravya "Contrast Enhancement Techniques using Histogram Equalization Methods on Color Images with Poor Lightning”, International Journal of Computer Science, Engineering and Applications (IJCSEA) Vol.3, No.4, August 2013.

[39] P. Suganya, S. Gayathri, N. Mohanapriya "Survey on Image Enhancement Techniques" International Journal of Computer Applications Technology and Research, Volume 2- Issue 5, 623 - 627, 2013.

[40] Biglari M, Mirzaei F, Ebrahimpour-Komeh H. Illumination invariant face recognition using SQI and weighted LBP histogram[C]. Pattern Recognition and Image Analysis (PRIA), 2013 First Iranian Conference on. IEEE, pp 1-7, 2013.

[41] E Krieger, VK Asari, and S Arigela. Color image enhancement of low-resolution images captured in extreme lighting conditions. In SPIE Sensing Technology and Applications, pages 91200Q91200Q. International Society for Optics and Photonics, 2014. 
[42] Anila, S., and N. Devarajan. "Preprocessing technique for face recognition applications under varying illumination conditions." Global Journal of Computer Science and Technology Volume 12, issue 11, Version 1, 2012.

[43] Nungsanginla Longkumer, Mukesh Kumar and Rohini Saxena "Contrast Enhancement Techniques using Histogram Equalization: A Survey" International Journal of Current Engineering and Technology, Vol.4, No.3, E-ISSN 2277 - 4106, P-ISSN 2347 - 5161 (June 2014).

[44] Ali M. Reza. "Realization of the Contrast Limited Adaptive Histogram Equalization (CLAHE) for Real-Time Image Enhancement." Journal of VLSI Signal Processing, vol. 38, pp 35-44, 2004.

(C) 2021 By AIRCC Publishing Corporation. This article is published under the Creative Commons Attribution (CC BY) license. 